\title{
Cubic Composite Sensor with Photodiodes for Tracking Solar Orientation
}

\author{
Yong-Nong Chang \\ Department of Electrical Engineering, National Formosa University, Yunlin County 63201, Taiwan \\ Correspondence should be addressed to Yong-Nong Chang; ynchang@nfu.edu.tw
}

Received 26 September 2013; Accepted 10 October 2013

Academic Editor: Liang-Wen Ji

Copyright (c) 2013 Yong-Nong Chang. This is an open access article distributed under the Creative Commons Attribution License, which permits unrestricted use, distribution, and reproduction in any medium, provided the original work is properly cited.

\begin{abstract}
A cubic composite solar sensor with photo diode is proposed for tracking the relative solar orientation. The proposed solar sensor composes of five photodiode detectors which are placed on the front, rear, left, right, and horizontal facets in a cubic body, respectively. The solar detectors placed on five facets can detect solar power of different facets. Based on the geometric coordinate transformation principle, the relationship equations of solar light orientation between measured powers with respect to various facets can be conducted. As a result, the solar orientation can be precisely achieved without needing any assistance of electronic compass and extra orientation angle corrector. Eventually, the relative solar light orientation, the elevation angle, and azimuth angle of the solar light can be measured precisely.
\end{abstract}

\section{Introduction}

Recently, the renewable energy has drawn great attention due to energy shortage. Many renewable energy researches are focused on solar power system [1-4]. For the solar system, a precise sensor is indispensable to tracking the sunlight orientation. Many studies have been focusing on solar tracking technique. However, there still exist some drawbacks on solar light tracking apparatus. Solar light tracker can, in general, be classified into mechanical and electronic category $[5,6]$. Mechanical solar tracker experiences much fault probability and maintenance cost, electronic solar tracker depends highly on electronic compass accompanied by perpetual calendar, and precise perpetual calendar heavily relies on complicated computation through the usage of internet, thus leading to inconvenience in application $[7,8]$. Therefore, to develop an electronic solar light tracker, without needing electronic compass and perpetual calendar, is essential to researchers and solar power applications.

An electronic solar light tracker can only be realized through the application of photo detecting devices. There are many photo devices such as photodiode, photo transistor, and resistive CdS components. The CdS components are passive components and usually cannot play the key role in a precise measurement. In this paper, the active photodiodes with wide measuring range and fast response and low dark current are applied as a solar sensor to measure the solar orientation.

The solar light orientation information should consist of the relative elevation angle and azimuth angle between solar source and photo detector. For collecting lights from all possible orientations, this paper employed five photodiodes to compose a cubic structure photo detector in the absence of electronic compass and perpetual calendar to accomplish an electronic solar light orientation tracking system. The composite sensor is composed of five photo diode detectors placed on five different facets: left, right, front, rear, and horizontal facets, to constitute a cubic structure solar light detector. By placing five detectors on five different facets, the sunlight orientation can be derived by geometric coordinate transformation technique.

There exists discrepancy in collecting power amounts for photo detectors on different facets. Moreover, the incident light on the photo diode comprises not only direct solar light but also diffuse light and scattered light [8]. The diffuse and scattered light power is also generally called undisciplined light power, denoted by $P_{\mathrm{U}}$. Therefore, the received solar power should be categorized precisely into power of direct incident solar light $P_{\mathrm{D}}$ and power of undisciplined incident solar light $P_{\mathrm{U}}$. Through the geometric transformation analysis and accurate evaluation formula, each collecting solar power 


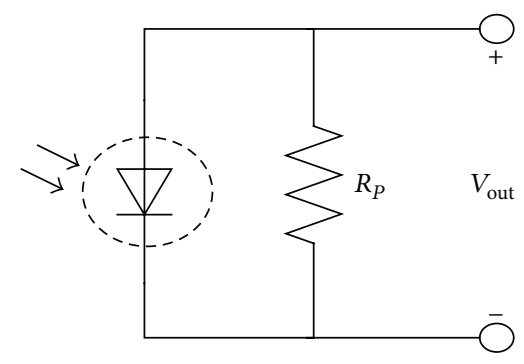

FIgURE 1: Photo diode applied circuit for detecting solar power.

on different facets can be divided into direct incident solar power $P_{\mathrm{D}}$ and undisciplined incident solar power $P_{\mathrm{U}}$, to accomplish the calculation of solar light orientation and solar polar powers on all facets.

In this paper, a novel light orientation sensor is proposed to track the solar orientation effectively. No expensive electronic components and no perpetual calendar are required. By appropriate computation based on different solar power quantities collected from five sensors, the sunlight direction can be determined. Also, the solar light orientation and power can easily be attained.

\section{Optimal Shunt Resistor Matching and Selection}

The ideal photo diode should generate an active current source which is proportional to the incident solar light. The total current through the photodiode is the sum of the dark current and the photo active current, so the dark current must be minimized to maximize the sensitivity of the device. In order to obtain the correct photo voltage, an appropriate shunt resistor $R_{P}$ should be shunted across the output terminals. The application circuit is shown in Figure 1 with external shunt resistor $R_{P}$; the photo voltage is generated at the two ends of the photo diode. The dark current has essential effects on the output voltage. Therefore, a calibration process for the shunt resistor $R_{P}$ is required.

The values of the output load resistor $R_{P}$. The adopted photo detector in this paper is produced by Hamamatsu Inc. with model code S1087-01. The parameters of the shunt resistor are assorted at $10 \Omega, 100 \Omega, 220 \Omega, 510 \Omega, 1 \mathrm{k} \Omega$, and $2 \mathrm{k} \Omega$.

The solar power is defined as $P_{\text {solar }}$, the output voltage is defined as $V_{\text {out }}$, and $S$ is defined as the sensitivity of the detector. In regard to an ideal photo power detector, $S$ should be kept with a constant quantity to maintain the linear relationship between sensing power and sensor output voltage as follows.

$$
P_{\text {Solar }}=\frac{V_{\text {out }}}{S}
$$

By shunting appropriate resistor $R_{P}$ in the output terminal, the solar power can be measured as shown in Figure 2, in case that ill condition occurs. The resistor value cannot be used to convert the current into voltage correctly. As shown in Figure 2, during the lower solar power period in the morning

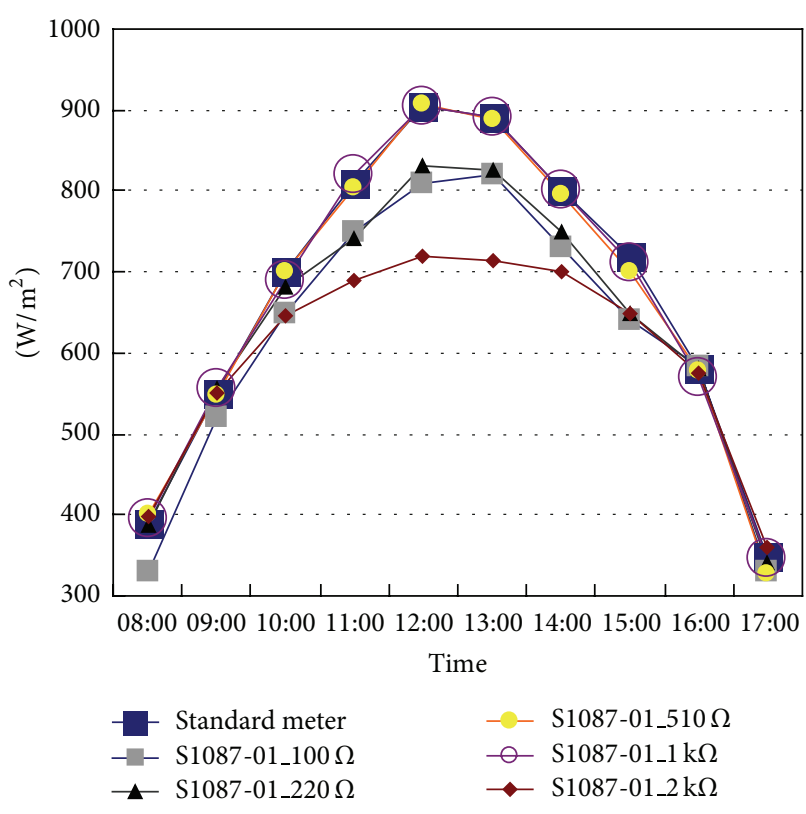

FIGURE 2: Optimal resistor matching and selection.

or evening periods, the solar power is roughly proportional to the photo current with resistor ranging from $100 \Omega$ to $220 \Omega$. However, the output power is slightly lower than the standard value during midday with direct sunlight. When the value is above $2 \mathrm{k} \Omega$, the solar power curve lies under the standard curve. When the resistor value is between $510 \Omega$ and $1 \mathrm{k} \Omega$, the output curve is inspected to be better fitting the theoretical solar power.

Since the photo diode has nonlinear equivalent characteristics as shown in Figure 2, an appropriate output resistor is required to be selected. When the sunlight is in either weak condition or strong condition, the solar power curve displays nonlinear phenomenon. When the value falls between $510 \Omega \sim 1 \mathrm{k} \Omega$, the curve has the best coincident condition with the standard curve. Considering that the identical solar power would generate identical output voltage, larger resistance will require less output current. Therefore, $1 \mathrm{k} \Omega$ resistor is selected to shunt with photo detector to enhance the linearity and precision of the proposed circuit.

In order to obtain the precise voltage conversion, an OPAMP circuit is designed to ensure the precise voltage gain for the five detectors. The calibration circuit of the detector is shown in Figure 3. The $0.01 \mu \mathrm{F}$ capacitor is connected across shunt resistor $R_{P}$ to eliminate noise.

\section{Geometric Coordinate Transformation and Formulation}

As designated in Figure 4, photo-detecting device is located at origin of the horizontal plane of $3 \mathrm{D}$ coordinate. The polar coordinate representation of position of light source is designated as $(r, \phi, \alpha)$ which results in the Cartesian 


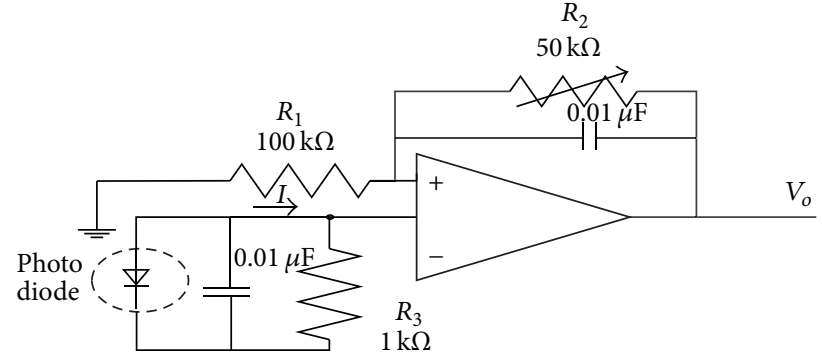

FIGURE 3: Detector calibration circuit.

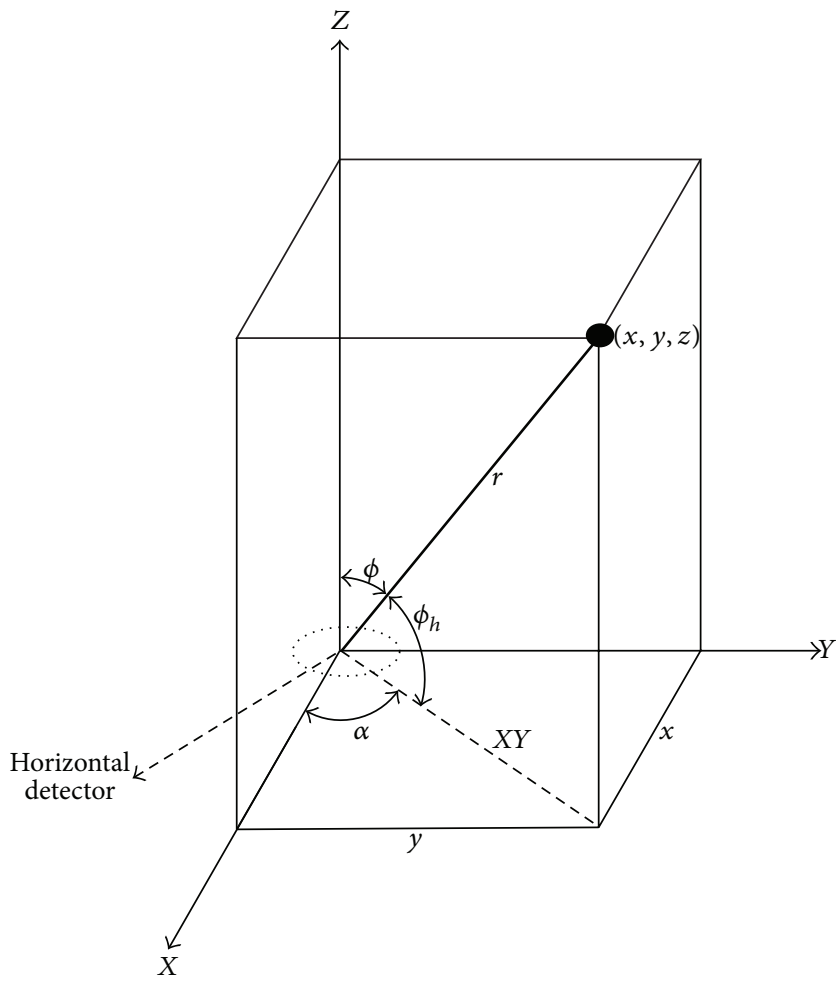

FIgURE 4: Geometric coordinate system.

coordinate and polar coordinate transformation relationship expressed as follows:

$$
\begin{gathered}
x=r \sin \phi \cos \alpha, \\
y=r \sin \phi \sin \alpha, \\
z=r \cos \phi,
\end{gathered}
$$

where $\phi$ is the light incident angle, $\alpha$ is the azimuth angle, and $r$ is the distance between light source and photo diode detector. Then, the elevation angle $\phi_{h}$ can be described as

$$
\phi_{h}=90^{\circ}-\phi .
$$

The orientation of sunlight is defined as elevation angle $\phi_{h}$ and azimuth angle $\alpha$ that means the orientation of the sunlight can be specified by formulation using the two angles: $\phi_{h}$ and $\alpha$.

For a 3D coordinate system, the rear direction is defined as the $X$ direction, the right direction is defined as the $Y$

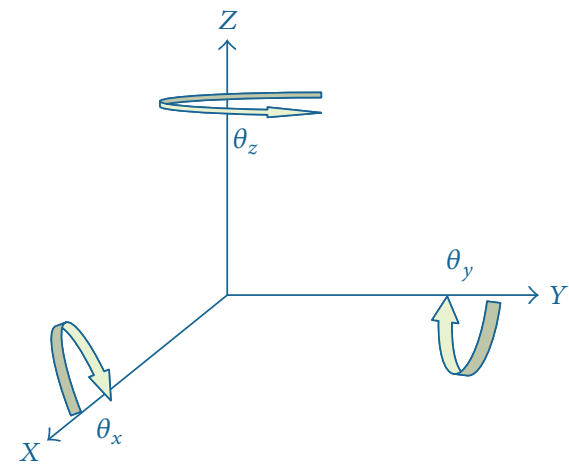

Figure 5: $\theta$ rotation along the $X-, Y$-, and $Z$-axis.

direction, and the normal vector direction of $X-Y$ horizontal plane is defined as the $Z$ direction, as indicated in Figure 4. The solar light incident angle $\phi$ can also be defined as the angle between the sunlight and the normal vector direction of horizontal plane. Thus, the horizontal detector collects direct light power $P_{\mathrm{HD}}$ which can be expressed as

$$
P_{\mathrm{HD}}=P_{\mathrm{D}} \cos \phi .
$$

The collected direct light power $P_{\mathrm{HD}}$ of horizontal detector is the multiplication of solar direct radiation power and cosine of incident angle $\phi$. Incident angle $\phi$ is defined as the angle between the normal vector of each facet with its corresponding incident light. Thus, when the horizontal detector rotates an angle $\theta$ along $X, Y$, and $Z$ axis, the associated incident angles of all facets will change accordingly. Figure 5 illustrates the rotational coordinate transformation by rotating along $Z$-axis with an angle $\theta_{Z}$ or along $X$-axis with $\theta_{x}$ or along $Y$-axis with $\theta_{y}$. By doing so, the original coordinate $(x, y$, $z)$ is transformed to a new coordinate $(x 1, y 1, z 1)$, and the expression can be written as follows.

(1) By rotating $\theta_{z}$ along $z$-axis, $x 1, y 1$, and $z 1$ can be obtained as follows

$$
\begin{aligned}
{\left[\begin{array}{l}
x 1 \\
y 1 \\
z 1
\end{array}\right] } & =\left[\begin{array}{ccc}
\cos \theta_{z} & -\sin \theta_{z} & 0 \\
\sin \theta_{z} & \cos \theta_{z} & 0 \\
0 & 0 & 1
\end{array}\right]\left[\begin{array}{l}
x \\
y \\
z
\end{array}\right] \\
& =r\left[\begin{array}{l}
\sin \phi \cos \alpha \cos \theta_{z}-\sin \phi \sin \alpha \sin \theta_{z} \\
\sin \phi \cos \alpha \sin \theta_{z}+\sin \phi \sin \alpha \cos \theta_{z} \\
\cos \phi
\end{array}\right] .
\end{aligned}
$$

In (5), new coordinate $z 1$ is identical with the original coordinate $z$. The angle between incident solar light and normal vector of detector plane of new coordinate still remains $\phi$; the projection on normal vector direction is $r \cos \phi$. Therefore, regardless of the rotating angle $\theta_{z}$, the incident of solar light keeps unchanged, that is, $\phi$. 
(2) By rotating $\theta_{x}$ along $x$-axis, $x 1, y 1$, and $z 1$ can be obtained as

$$
\begin{aligned}
{\left[\begin{array}{l}
x 1 \\
y 1 \\
z 1
\end{array}\right] } & =\left[\begin{array}{ccc}
1 & 0 & 0 \\
0 & \cos \theta_{x} & -\sin \theta_{x} \\
0 & \sin \theta_{x} & \cos \theta_{x}
\end{array}\right]\left[\begin{array}{l}
x \\
y \\
z
\end{array}\right] \\
& =r\left[\begin{array}{l}
\sin \phi \cos \alpha \\
\sin \phi \sin \alpha \cos \theta_{x}-\cos \phi \sin \theta_{x} \\
\sin \phi \sin \alpha \sin \theta_{x}+\cos \phi \sin \theta_{x}
\end{array}\right] .
\end{aligned}
$$

The projection component of solar light on the normal vector of new coordinate plane $z 1$ deviates a significant quantity from the original projection value $z$, so does the incident angle.

(3) By rotating $\theta_{y}$ along $y$-axis, $x 1, y 1$, and $z 1$ can be obtained as

$$
\begin{aligned}
{\left[\begin{array}{l}
x 1 \\
y 1 \\
z 1
\end{array}\right] } & =\left[\begin{array}{ccc}
\cos \theta_{y} & 0 & \sin \theta_{y} \\
0 & 1 & 0 \\
-\sin \theta_{y} & 0 & \cos \theta_{y}
\end{array}\right]\left[\begin{array}{l}
x \\
y \\
z
\end{array}\right] \\
& =r\left[\begin{array}{l}
\sin \phi \cos \alpha \cos \theta_{y}+\cos \phi \sin \theta_{y} \\
\sin \phi \sin \alpha \\
-\sin \phi \cos \alpha \sin \theta_{y}+\cos \phi \cos \theta_{y}
\end{array}\right] .
\end{aligned}
$$

Like that of rotation along $X$-axis, the projection of incident light on normal vector of new coordinate plane $z 1$ deviates noticeably from original one, $z$. It implies that incident angle changes with rotation angle $\theta_{y}$.

After installing five detectors on five different facets, the cubic composite sensor is shown in Figure 6, Aside from the horizontal facet detector, the other four detectors can be looked upon as those coordinates transformed from the horizontal one by rotating either along $X$-axis or $Y$-axis with $\pm 90^{\circ}$. The coordinate transformation can be derived in the following section.

\section{Cubic Composite Sensor}

The relationship between direct incident solar power $P_{\mathrm{D}}$, undisciplined power $P_{\mathrm{U}}$, the light incident angle $\phi$, and the azimuth angle $\alpha$ can be discussed in this section. Since the five detectors are located on five different facets of a cubic box, the coordinate transformation relationships between five facets will be discussed below.

(1) The horizontal $(\mathrm{H})$ detector is located on the facet which $\theta_{x}=0^{\circ}$ along $z$-axis. Therefore, the transformed orientation coordinate of the sunlight and corresponding collecting power can be derived as

$$
\begin{aligned}
{\left[\begin{array}{l}
x 1 \\
y 1 \\
z 1
\end{array}\right] } & =r\left[\begin{array}{l}
\sin \phi \cos \alpha \cos 0^{\circ}-\sin \phi \sin \alpha \sin 0^{\circ} \\
\sin \phi \cos \alpha \sin 0^{\circ}+\sin \phi \sin \alpha \cos 0^{\circ} \\
\cos \phi
\end{array}\right] \\
& =r\left[\begin{array}{l}
\sin \phi \cos \alpha \\
\sin \phi \sin \alpha \\
\cos \phi
\end{array}\right]=\left[\begin{array}{l}
x \\
y \\
z
\end{array}\right] .
\end{aligned}
$$

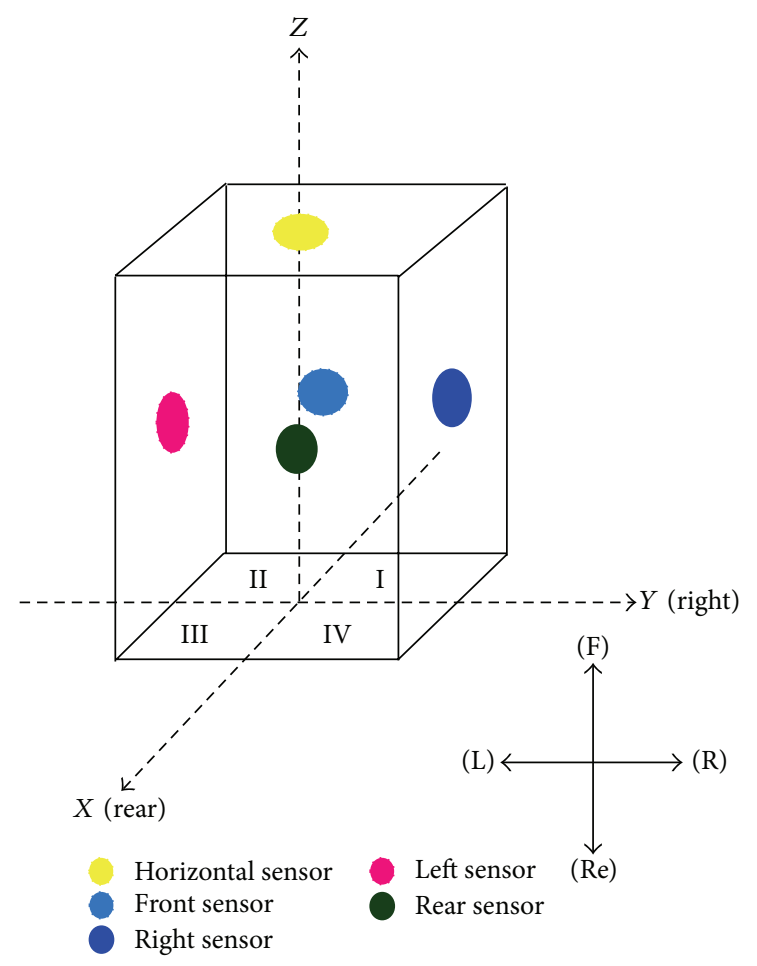

FIGURE 6: Cubic sensors model in a 3D coordinate system.

The normal vector of horizontal plane $z 1$ is the same with the original normal vector $z$; the incident angle is kept unchanged, that is, $\phi_{\mathrm{H}}=\phi$,

$$
\begin{gathered}
\phi_{\mathrm{H}}=\phi \\
P_{\mathrm{H}}=P_{\mathrm{D}} \cos \phi+P_{\mathrm{UH}}=P_{\mathrm{D}} \cos \phi_{\mathrm{H}}+P_{\mathrm{UH}}
\end{gathered}
$$

The collected power $P_{\mathrm{H}}$ on the horizontal detector can be expressed as (10). Wherein, $P_{\mathrm{D}}$ is the maximum direct incident solar power and $P_{\mathrm{UH}}$ is the power caused by light diffusion and scattering.

(2) The right sensor is located on facet which $\theta_{x}=90^{\circ}$ along $x$-axis. Therefore, the orientation of the sunlight can be derived as

$$
\begin{aligned}
{\left[\begin{array}{l}
x 1 \\
y 1 \\
z 1
\end{array}\right] } & =r\left[\begin{array}{c}
\sin \phi \cos \alpha \\
\sin \phi \sin \alpha \cos 90^{\circ}-\cos \phi \sin 90^{\circ} \\
\sin \phi \sin \alpha \sin 90^{\circ}+\cos \phi \cos 90^{\circ}
\end{array}\right] \\
& =r\left[\begin{array}{c}
\sin \phi \cos \alpha \\
-\cos \phi \\
\sin \phi \sin \alpha
\end{array}\right]=\left[\begin{array}{c}
x \\
-z \\
y
\end{array}\right]
\end{aligned}
$$

As shown in (11), right facet detector is equivalent to rotating horizontal facet by $\theta_{x}=90^{\circ}$. The normal vector of right plane is directed toward $Z$-axis which is the positive $Y$-axis direction of horizontal facet. The light incident angle $\phi_{\mathrm{R}}$ is related to original incident 
angle $\phi$ by (12). Next, the collected power on the right facet detector $P_{\mathrm{R}}$ is written as

$$
\begin{gathered}
\phi_{\mathrm{R}}=\cos ^{-1}(\sin \phi \sin \alpha) \\
P_{\mathrm{R}}=P_{\mathrm{D}} \sin \phi \sin \alpha+P_{\mathrm{UR}}=P_{\mathrm{D}} \cos \phi_{\mathrm{R}}+P_{\mathrm{UR}}
\end{gathered}
$$

where $P_{\mathrm{UR}}$ is the undisciplined light power collected by right detector.

(3) The left sensor is located on facet which $\theta_{x}=-90^{\circ}$ along $x$-axis. Therefore, the orientation of the sunlight can be derived as

$$
\begin{aligned}
{\left[\begin{array}{l}
x 1 \\
y 1 \\
z 1
\end{array}\right] } & =r\left[\begin{array}{c}
\sin \phi \cos \alpha \\
\sin \phi \sin \alpha \cos \left(-90^{\circ}\right)-\cos \phi \sin \left(-90^{\circ}\right) \\
\sin \phi \sin \alpha \sin \left(-90^{\circ}\right)+\cos \phi \cos \left(-90^{\circ}\right)
\end{array}\right] \\
= & r\left[\begin{array}{c}
\sin \phi \cos \alpha \\
\cos \phi \\
-\sin \phi \sin \alpha
\end{array}\right]=\left[\begin{array}{c}
x \\
z \\
-y
\end{array}\right] .
\end{aligned}
$$

As shown in (14), left facet detector is equivalent to rotating horizontal facet by $\theta_{x}=-90^{\circ}$. The normal vector of left plane is directed toward the negative $Y$ axis direction of horizontal facet. The light incident angle $\phi_{\mathrm{L}}$ is related to original incident angle $\phi$ by (15). More, the collected power on the right facet detector $P_{\mathrm{L}}$ is written as

$$
\begin{gathered}
\phi_{\mathrm{L}}=\cos ^{-1}(-\sin \phi \sin \alpha), \\
P_{\mathrm{L}}=-P_{\mathrm{D}} \sin \phi \cos \alpha+P_{\mathrm{UL}}=P_{\mathrm{D}} \cos \phi_{\mathrm{L}}+P_{\mathrm{UL}}
\end{gathered}
$$

where $P_{\mathrm{UL}}$ is the undisciplined light power collected by left detector.

(4) The front sensor is located on facet which $\theta_{y}=90^{\circ}$ along $y$-axis. Therefore, the orientation of the sunlight can be derived as

$$
\begin{aligned}
{\left[\begin{array}{l}
x 1 \\
y 1 \\
z 1
\end{array}\right] } & =r\left[\begin{array}{c}
\sin \phi \cos \alpha \cos 90^{\circ}+\cos \phi \sin 90^{\circ} \\
\sin \phi \sin \alpha \\
-\sin \phi \cos \alpha \sin 90^{\circ}+\cos \phi \cos 90^{\circ}
\end{array}\right] \\
& =r\left[\begin{array}{c}
\cos \phi \\
\sin \phi \sin \alpha \\
-\sin \phi \cos \alpha
\end{array}\right]=\left[\begin{array}{c}
z \\
y \\
-x
\end{array}\right] .
\end{aligned}
$$

As expressed in (16), front facet detector is equivalent to rotating horizontal facet by $\theta_{y}=90^{\circ}$. The normal vector of front plane is directed toward the negative $X$-axis direction of horizontal facet. The light incident angle $\phi_{\mathrm{F}}$ is related to original incident angle $\phi$ by (18). Next, the collected power on the front facet detector $P_{F}$ is written as

$$
\begin{gathered}
\phi_{\mathrm{F}}=\cos ^{-1}(-\sin \phi \cos \alpha), \\
P_{\mathrm{F}}=-P_{\mathrm{D}} \sin \phi \cos \alpha+P_{\mathrm{UF}}=P_{\mathrm{D}} \cos \phi_{\mathrm{F}}+P_{\mathrm{UF}}
\end{gathered}
$$

where $P_{\mathrm{UF}}$ is the undisciplined light power collected by front detector.

(5) The rear sensor is located on facet which $\theta_{y}=-90^{\circ}$ along $y$-axis. Therefore, the orientation of the sunlight can be derived as

$$
\begin{aligned}
{\left[\begin{array}{l}
x 1 \\
y 1 \\
z 1
\end{array}\right] } & =r\left[\begin{array}{c}
\sin \phi \cos \alpha \cos \left(-90^{\circ}\right)+\cos \phi \sin \left(-90^{\circ}\right) \\
\sin \phi \sin \alpha \\
-\sin \phi \cos \alpha \sin \left(-90^{\circ}\right)+\cos \phi \cos \left(-90^{\circ}\right)
\end{array}\right] \\
& =r\left[\begin{array}{c}
-\cos \phi \\
\sin \phi \sin \alpha \\
\sin \phi \cos \alpha
\end{array}\right]=\left[\begin{array}{c}
-z \\
y \\
x
\end{array}\right] .
\end{aligned}
$$

As shown in (20), rear facet detector is equivalent to rotating horizontal facet by $\theta_{y}=-90^{\circ}$. The normal vector of rear plane is directed toward the positive $X$ axis direction of horizontal facet. The light incident angle $\phi_{\operatorname{Re}}$ is related to original incident angle $\phi$ by (21). Next, the collected power on the rear facet detector $P_{\mathrm{Re}}$ is written as

$$
\begin{gathered}
\phi_{\mathrm{Re}}=\cos ^{-1}(\sin \phi \cos \alpha) \\
P_{\mathrm{Re}}=P_{\mathrm{D}} \sin \phi \cos \alpha+P_{\mathrm{URe}}=P_{\mathrm{D}} \cos \phi_{\mathrm{Re}}+P_{\mathrm{URe}}
\end{gathered}
$$

where $P_{\mathrm{URe}}$ is the undisciplined light power collected by rear detector.

Figure 6 defines incident quadrant of the solar light. The assessing criteria of incident quadrant are based on the collecting light power of five facets $P_{\mathrm{H}}, P_{\mathrm{R}}, P_{\mathrm{L}}, P_{\mathrm{F}}$, and $P_{\mathrm{Re}}$, respectively. By comparing the quantities of the collecting light power, the criteria are described as follows.

(1) When the $P_{\mathrm{R}}>P_{\mathrm{L}}$ and $P_{\mathrm{F}}>P_{\mathrm{Re}}$, the sunlight comes from the first quadrant.

(2) When the $P_{\mathrm{L}}>P_{\mathrm{R}}$ and $P_{\mathrm{F}}>P_{\mathrm{Re}}$, the sunlight comes from the second quadrant.

(3) When the $P_{\mathrm{L}}>P_{\mathrm{R}}$ and $P_{\mathrm{Re}}>P_{\mathrm{F}}$, the sunlight comes from the third quadrant.

(4) When the $P_{\mathrm{R}}>P_{\mathrm{L}}$ and $P_{\mathrm{Re}}>P_{\mathrm{F}}$, the sunlight comes from the fourth quadrant.

Once the solar light incident quadrant is determined, the solar power orientation and power information can be obtained by substituting (8) in to (22). When the sunlight is incident to the fourth quadrant, the relationships of $\phi_{\mathrm{F}}, \phi_{\mathrm{R}}, \alpha$, and $P_{\mathrm{D}}$ can be conducted from (12) to (15) to arrive at (23) as follows:

$$
\begin{gathered}
P_{\mathrm{D}} \cos \phi_{\mathrm{Re}}=P_{\mathrm{D}} \sin \phi \cos \alpha, \\
P_{\mathrm{D}} \cos \phi_{\mathrm{R}}=P_{\mathrm{D}} \sin \phi \sin \alpha .
\end{gathered}
$$

Since the incident light falls on the fourth quadrant, the direct light cannot reach front and left facets. The collecting solar power can merely come from the undisciplined light power. Consequently, the undisciplined light power of (23) 
TABLE 1: Solar power with respect to five detectors (unit: $\left(\mathrm{W} / \mathrm{m}^{2}\right)$ ).

\begin{tabular}{ccccccc}
\hline Time & \multicolumn{4}{c}{ Facet } & \multicolumn{2}{c}{ Light incident quadrant } \\
& $P_{\mathrm{R}}$ & $P_{\mathrm{Re}}$ & $P_{\mathrm{L}}$ & $P_{\mathrm{F}}$ & $P_{\mathrm{H}}$ & I \\
\hline $08: 00$ & 454.7 & 105.1 & 92.4 & 164.1 & 220.2 & $\mathrm{I}$ \\
$09: 00$ & 526.7 & 135.3 & 113.3 & 170.9 & 390.2 & $\mathrm{I}$ \\
$10: 00$ & 467.0 & 128.9 & 120.4 & 172.5 & 552.8 & $\mathrm{I}$ \\
$11: 00$ & 439.9 & 136.4 & 127.4 & 149.0 & 703.0 & IV \\
$12: 00$ & 267.5 & 130.0 & 121.1 & 118.6 & 815.9 & III \\
$13: 00$ & 91.1 & 111.9 & 103.7 & 92.6 & 953.9 & III \\
$14: 00$ & 141.4 & 147.8 & 317.3 & 112.7 & 860.4 & II \\
$15: 00$ & 143.3 & 136.7 & 452.9 & 148.8 & 691.6 & II \\
$16: 00$ & 168.6 & 158.4 & 511.5 & 198.2 & 582.9 &
\end{tabular}

can be roughly substituted by collected power quantity of adjacent facets. In other words, the direct solar light falling on the fourth quadrant comes from right and rear directions; the undisciplined light power on the rear facet $P_{\mathrm{URe}}$ is approximated by left facet light power $P_{\mathrm{L}}$; the undisciplined light power on the right facet $P_{\mathrm{UR}}$ is approximated by front facet light power $P_{\mathrm{F}}$.

From (23), the $\alpha$ angle can be derived as follows

$$
\frac{P_{\mathrm{RD}}}{P_{\mathrm{ReD}}}=\frac{P_{\mathrm{D}} \sin \phi \sin \alpha}{P_{\mathrm{D}} \sin \phi \cos \alpha}=\frac{\sin \alpha}{\cos \alpha}=\tan \alpha \Longrightarrow \alpha=\tan ^{-1} \frac{P_{\mathrm{RD}}}{P_{\mathrm{ReD}}} .
$$

From (10) and (22), the $\phi$ angle can be derived as follows

$$
\frac{P_{\mathrm{ReD}}}{P_{\mathrm{HD}}}=\frac{\sin \phi \cos \alpha}{\cos \phi}=\tan \phi \cos \alpha \Longrightarrow \phi=\tan ^{-1} \frac{P_{\mathrm{ReD}}}{P_{\mathrm{HD}} \cos \alpha} \text {. }
$$

After the $\phi$ angle is decided, the $P_{\mathrm{D}}$ can be derived as

$$
P_{\mathrm{D}}=\frac{P_{\mathrm{HD}}}{\cos \phi} \text {. }
$$

The incident quadrant of incident light is changing with time. Similar derivation can be used to obtain the solar light orientation and power information.

\section{Experimental Results}

In order to verify the formulation, an experimental measurement was performed on June 02, 2013. Table 1 lists the collecting solar power of all facet detectors on the hour.

In Table 1, to take data at 12:00 A.M. as an example, the cubic sensor receives the incident light from the fourth quadrant, the detectors on the right, rear, and horizontal facets can absorb power of direct incident solar light $P_{\mathrm{D}}$. However, the left and front facets can only absorb power of undisciplined incident solar light $P_{\mathrm{U}}$. Therefore, the collecting solar power on the left detector $P_{\mathrm{L}}$ is near the undisciplined power $P_{\mathrm{UL}}$. Also, the collecting solar power on the front detector $P_{\mathrm{F}}$ can be approximated by the undisciplined power $P_{\mathrm{UF}}$. The collected undisciplined power on the horizontal
TABLE 2: Undisciplined incident solar light power with respect to five detectors (unit: $\left(\mathrm{W} / \mathrm{m}^{2}\right)$ ).

\begin{tabular}{ccccccc}
\hline \multirow{2}{*}{ Time } & \multicolumn{3}{c}{ Facet } & \multicolumn{2}{c}{ Light incident quadrant } \\
& $P_{\mathrm{UR}}$ & $P_{\mathrm{URe}}$ & $P_{\mathrm{UL}}$ & $P_{\mathrm{UF}}$ & $P_{\mathrm{UH}}$ & $\mathrm{I}$ \\
\hline $08: 00$ & 105.1 & 105.1 & 92.4 & 92.4 & 98.7 & $\mathrm{I}$ \\
$09: 00$ & 135.3 & 135.3 & 113.3 & 113.3 & 124.3 & $\mathrm{I}$ \\
$10: 00$ & 128.9 & 128.9 & 120.4 & 120.4 & 125.7 & $\mathrm{I}$ \\
$11: 00$ & 136.4 & 136.4 & 127.4 & 127.4 & 131.8 & IV \\
$12: 00$ & 118.6 & 121.1 & 121.1 & 118.6 & 119.9 & III \\
$13: 00$ & 91.1 & 91.1 & 92.6 & 92.6 & 91.8 & III \\
$14: 00$ & 141.4 & 141.4 & 112.7 & 112.7 & 127.1 & II \\
$15: 00$ & 143.3 & 136.7 & 136.7 & 143.3 & 140.0 & II \\
$16: 00$ & 168.6 & 158.4 & 158.4 & 168.6 & 163.5 &
\end{tabular}

TAble 3: Direct incident solar light power with respect to five detectors (unit: $\left(\mathrm{W} / \mathrm{m}^{2}\right)$ ).

\begin{tabular}{ccccccc}
\hline Time & \multicolumn{5}{c}{ Facet } & \multicolumn{2}{c}{ Light incident quadrant } \\
& $P_{\mathrm{RD}}$ & $P_{\mathrm{ReD}}$ & $P_{\mathrm{LD}}$ & $P_{\mathrm{FD}}$ & $P_{\mathrm{HD}}$ & $\mathrm{I}$ \\
\hline $08: 00$ & 349.6 & 0 & 0 & 71.7 & 121.5 & $\mathrm{I}$ \\
$09: 00$ & 391.4 & 0 & 0 & 57.6 & 265.9 & $\mathrm{I}$ \\
$10: 00$ & 338.1 & 0 & 0 & 52.1 & 427.1 & $\mathrm{I}$ \\
$11: 00$ & 303.5 & 0 & 0 & 21.6 & 571.2 & IV \\
$12: 00$ & 148.9 & 8.9 & 0 & 0 & 696.0 & III \\
$13: 00$ & 0 & 20.8 & 11.1 & 0 & 862.1 & III \\
$14: 00$ & 0 & 6.4 & 204.6 & 0 & 733.3 & II \\
$15: 00$ & 0 & 0 & 316.2 & 5.5 & 551.6 & II \\
$16: 00$ & 0 & 0 & 353.1 & 29.6 & 419.4 &
\end{tabular}

detector equals around the average of these two quantities. Besides, any detector, with direct incident solar power, possesses collected undisciplined solar power which is similar with the power collecting environment of adjacent facet detector. Thus, the collected undisciplined solar power can be substituted by collecting solar power on adjacent facet detector. It means that $P_{\mathrm{URe}}$ equals $P_{\mathrm{L}}$ and $P_{\mathrm{UR}}$ equals $P_{\mathrm{UF}}$. Table 2 lists the undisciplined powers of each facet on the hour. Table 3 lists the direct incident solar power of each detector which is obtained by deducting undisciplined power from measured power. Therefore, the listed quantities in Table 1 are equal to the summation of those corresponding entities in Tables 2 and 3.

Based on the direct incident solar light power on five facets listed in Table 3, the orientation angles at different times can be evaluated. Taking the measured power at 12:00 A.M. on the selected day as an example, the azimuth angle and elevation angle are calculated and obtained as follows.

According to (24), it arrives at $\alpha$ :

$$
\alpha=\tan ^{-1} \frac{P_{\mathrm{RD}}}{P_{\mathrm{ReD}}}=\tan ^{-1} \frac{148.9}{8.9}=86.58^{\circ} .
$$


TABLE 4: Azimuth angles comparison between theoretical and computed values.

\begin{tabular}{cccc}
\hline Time & $\begin{array}{c}\text { Theoretical } \\
\text { azimuth angles } \\
\alpha\end{array}$ & $\begin{array}{c}\text { Computed } \\
\text { azimuth angles } \\
\alpha\end{array}$ & Error \\
\hline $08: 00$ & $105.14^{\circ}$ & $101.59^{\circ}$ & $3.55^{\circ}$ \\
$09: 00$ & $100.82^{\circ}$ & $98.37^{\circ}$ & $2.45^{\circ}$ \\
$10: 00$ & $96.72^{\circ}$ & $98.76^{\circ}$ & $-2.04^{\circ}$ \\
$11: 00$ & $92.30^{\circ}$ & $94.07^{\circ}$ & $-1.77^{\circ}$ \\
$12: 00$ & $85.55^{\circ}$ & $86.58^{\circ}$ & $-1.03^{\circ}$ \\
$13: 00$ & $331.06^{\circ}$ & $331.91^{\circ}$ & $-0.85^{\circ}$ \\
$14: 00$ & $273.15^{\circ}$ & $271.79^{\circ}$ & $1.36^{\circ}$ \\
$15: 00$ & $267.02^{\circ}$ & $269.00^{\circ}$ & $-1.98^{\circ}$ \\
$16: 00$ & $262.70^{\circ}$ & $265.21^{\circ}$ & $-2.51^{\circ}$ \\
\hline
\end{tabular}

TABLE 5: Elevation angles comparison between theoretical and computed values.

\begin{tabular}{cccc}
\hline Time & $\begin{array}{c}\text { Theoretical } \\
\text { elevation angles } \\
\phi_{h}\end{array}$ & $\begin{array}{c}\text { Computed } \\
\text { elevation angles } \\
\phi_{h}\end{array}$ & Error \\
\hline $08: 00$ & $22.61^{\circ}$ & $18.80^{\circ}$ & $3.81^{\circ}$ \\
$09: 00$ & $36.00^{\circ}$ & $33.91^{\circ}$ & $2.09^{\circ}$ \\
$10: 00$ & $49.57^{\circ}$ & $51.31^{\circ}$ & $-1.74^{\circ}$ \\
$11: 00$ & $63.25^{\circ}$ & $61.96^{\circ}$ & $1.29^{\circ}$ \\
$12: 00$ & $76.98^{\circ}$ & $77.90^{\circ}$ & $-0.92^{\circ}$ \\
$13: 00$ & $88.14^{\circ}$ & $88.43^{\circ}$ & $-0.29^{\circ}$ \\
$14: 00$ & $75.20^{\circ}$ & $74.40^{\circ}$ & $0.80^{\circ}$ \\
$15: 00$ & $61.48^{\circ}$ & $60.17^{\circ}$ & $1.31^{\circ}$ \\
$16: 00$ & $47.81^{\circ}$ & $49.81^{\circ}$ & $-2.00^{\circ}$ \\
\hline
\end{tabular}

Using (25), incident angle $\phi$ can be obtained as

$$
\phi=\tan ^{-1} \frac{P_{\mathrm{RD}}}{P_{\mathrm{HD}} \sin \alpha}=12.1^{\circ} .
$$

Elevation angle is the complimentary angle of incident angle; thus,

$$
\phi_{h}=90-\phi=77.9^{\circ} .
$$

The theoretical azimuth angle and the computed azimuth angle are shown in Table 4, and the theoretical elevation angle and the computed elevation angle are shown in Table 5, respectively. Theoretical azimuth angles and elevation angles are obtained from perpetual calendar.

In this paper, the light diffusion factor is neglected and not taken into the formulation. Only simple constant offset value is used to present the diffusion power. In Tables 4 and 5 , the elevation angles and azimuth angles are quoted and calculated. In Table 4, by comparing the theoretical azimuth angles and computed azimuth angles, the errors are kept below $3.55^{\circ}$. In Table 5 , by comparing the theoretical elevation angles and the computed elevation angles, the errors are also kept below $3.81^{\circ}$ which is within acceptable range.

\section{Conclusion}

This paper has successfully proposed a cubic composite sensor with five sensors placing on five different facets. By coordinate transformation and detailed formulation, the azimuth angles and elevation angles for different sunlight orientations can be derived. Accordingly the sunlight orientation can be determined. No electronic compass and CdS resistors are required in the mechanism. Results show that the proposed method has precise solar tracking performance. This proves the validity of the proposed formulation to estimate the sunlight orientation and solar power. Besides, the solar light power can also be measured and provide valuable information for the solar tracking system.

\section{Conflict of Interests}

The author declares that there is no conflict of interests regarding the publication of this paper.

\section{Acknowledgment}

The author would like to acknowledge the financial support of the National Science Council of Taiwan, through grant number 102-2622-E-150-005-CC3.

\section{References}

[1] F. Katiraei and J. R. Agüero, "Solar PV integration challenges," IEEE Power and Energy Magazine, vol. 9, no. 3, pp. 62-71, 2011.

[2] A. Pandey, N. Dasgupta, and A. K. Mukerjee, "A simple singlesensor MPPT solution," IEEE Transactions on Power Electronics, vol. 22, no. 2, pp. 698-700, 2007.

[3] H. Hayami, M. Nakamura, and K. Yoshioka, "The life cycle $\mathrm{CO}_{2}$ emission performance of the DOE/NASA solar power satellite system: a comparison of alternative power generation systems in Japan," IEEE Transactions on Systems, Man, and Cybernetics C, vol. 35, pp. 391-400, 2005.

[4] J. Chadjivassiliadis, "Solar photovoltaic and wind power in Greece, physical science, measurement and instrumentation, management and education-reviews," IEE Proceedings A, vol. 134, pp. 457-463, 2008.

[5] M. H. Rahman and S. Yamashiro, "Novel distributed power generating system of PV-ECaSS using solar energy estimation," IEEE Transactions on Energy Conversion, vol. 22, no. 2, pp. 358367, 2007.

[6] S. Seme, G. Štumberger, and J. Voršič, "Maximum efficiency trajectories of a two-axis sun tracking system determined considering tracking system consumption," IEEE Transactions on Power Electronics, vol. 26, no. 4, pp. 1280-1290, 2011.

[7] A. Dolara, F. Grimaccia, S. Leva, M. Mussetta, R. Faranda, and M. Gualdoni, "Performance analysis of a single-axis tracking PV system," IEEE Journal of Photovoltaics, vol. 2, pp. 524-531, 2013.

[8] F. Zhang, K. Thanapalan, A. Procter, S. Carr, and J. Maddy, "Adaptive hybrid maximum power point tracking method for a photovoltaic system," IEEE Transactions on Energy Conversion, vol. 28, pp. 353-360, 2013. 

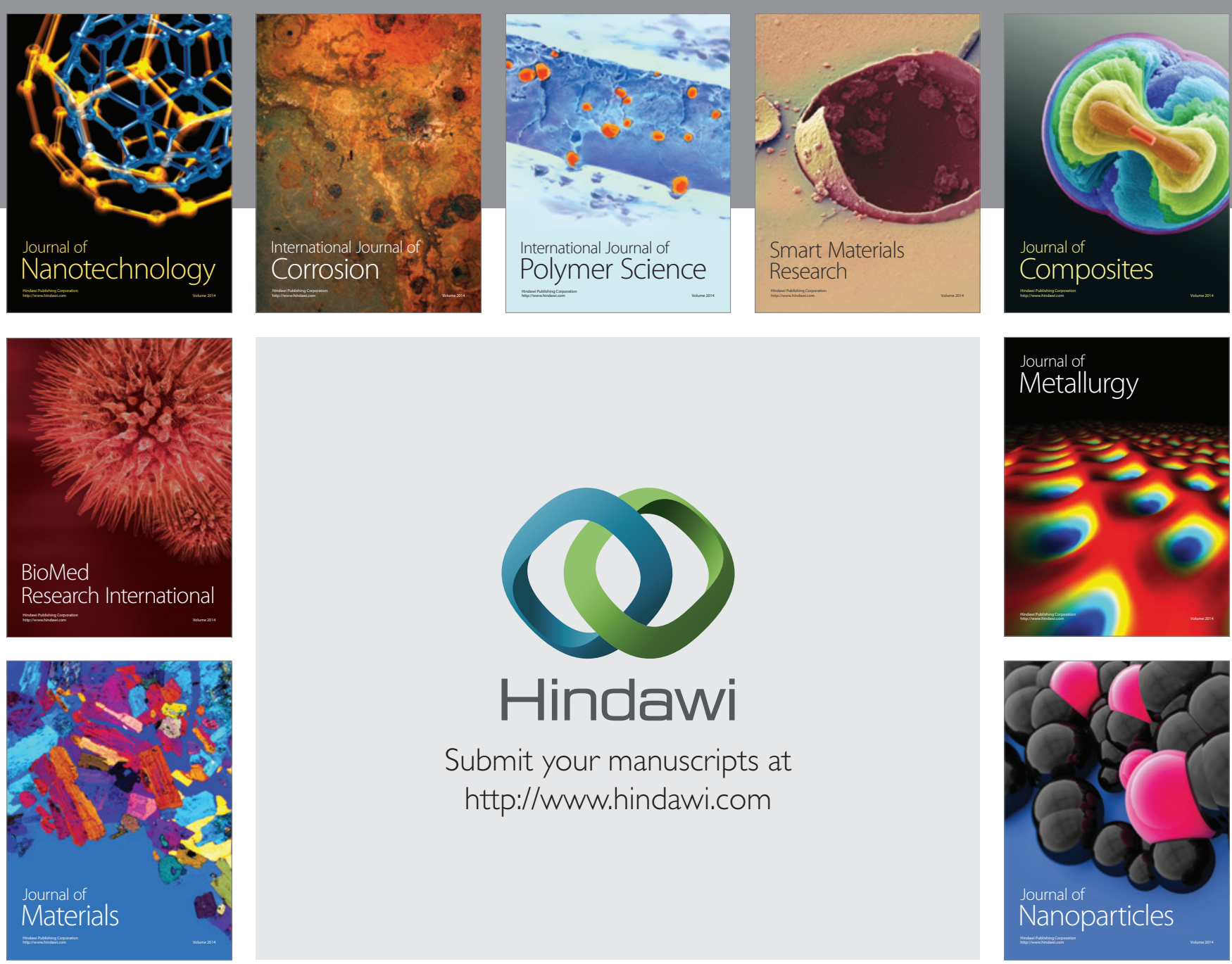

Submit your manuscripts at http://www.hindawi.com
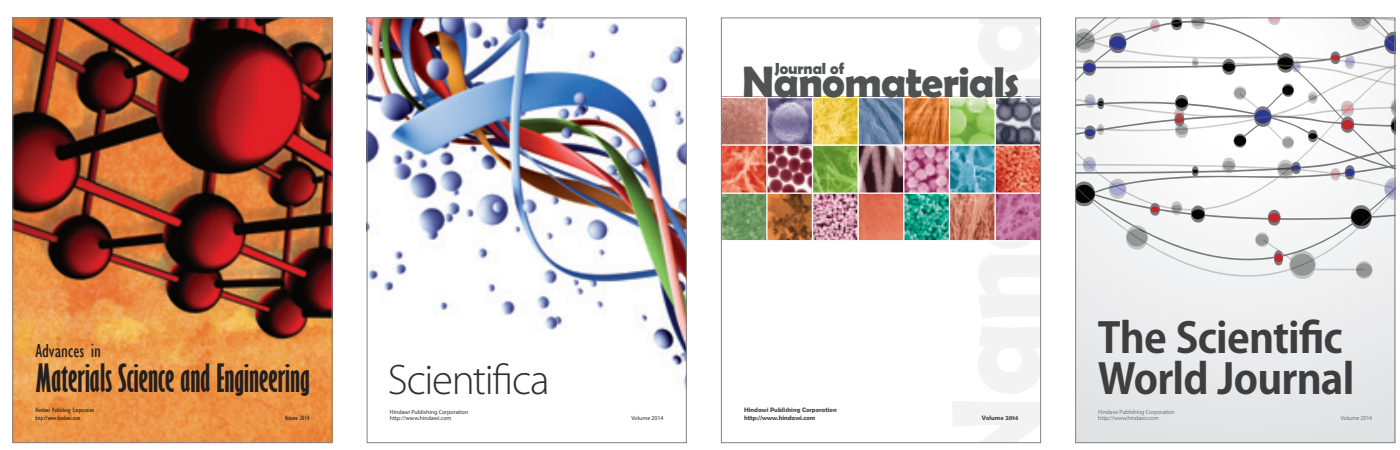

\section{The Scientific World Journal}
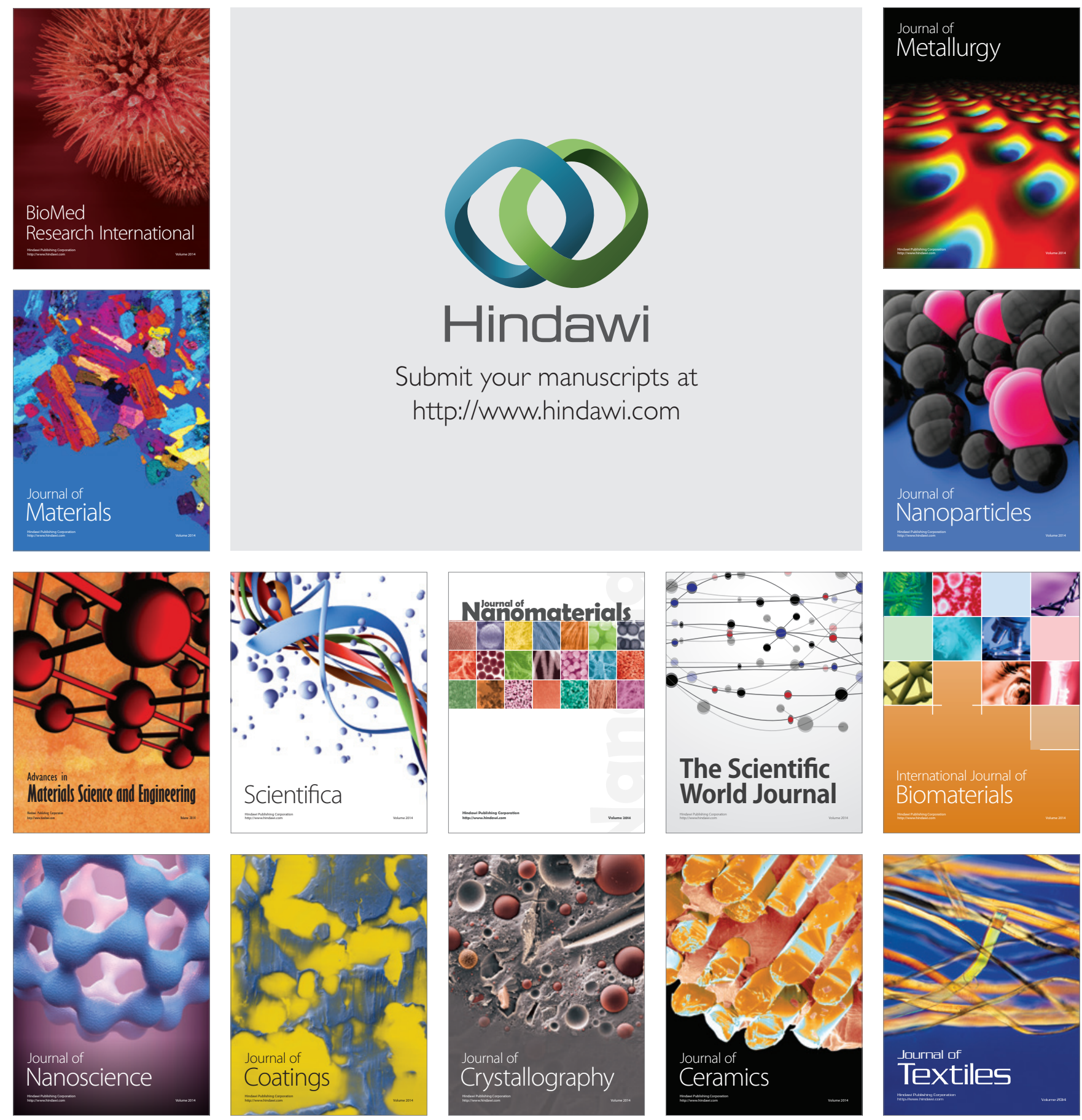